\title{
Electron-Beam-Damage in Metal Organic Frameworks in the TEM
}

\author{
Supriya Ghosh $^{1 *}$, Prashant Kumar ${ }^{1,2}$, Sabrina Conrad ${ }^{1}$, Michael Tsapatsis ${ }^{1,3}$, K. Andre Mkhoyan ${ }^{1}$. \\ 1. Department of Chemical Engineering and Material Science, University of Minnesota, Minneapolis, \\ MN. \\ 2. Department of Chemical Engineering, University of Michigan, Ann Arbor, MI. \\ 3. Department of Chemical and Biomolecular Engineering, Johns Hopkins University, Baltimore, MD. \\ * Corresponding author: ghosh115@umn.edu
}

Metal Organic Frameworks (MOFs) are hybrid inorganic-organic porous materials that are gaining interest for applications in catalysis, gas-storage, and gas-separations [1]. The visualization of the atomic structure of MOFs is necessary to study their crystal structure, interfaces and defects in them to understand the mass-transport and sorption characteristics [2]. Investigation of the MOF atomic structure in the TEM is challenging due to unusually high electron beam-sensitivity of the material [3]. A systematic study of the electron-beam-damage mechanisms occurring in MOFs is needed to enable characterization of MOFs in the TEM. Damage in the TEM occurs due to inelastic scattering of probe electrons with the atoms of the sample, leading to knock-on damage, radiolysis and charging in the specimen [4]. In this study, we examine one of the common MOFs, Zeolitic Imidazolate Framework, ZIF-8, to understand the dominating damage mechanism and evaluate the damage cross-sections for radiolysis $\left(\sigma_{\mathrm{r}}\right)$ and knock-on $\left(\sigma_{\mathrm{k}}\right)$. The results will be compared to the damage mechanisms in zeolites, which are also porous beam-sensitive materials [5].

ZIF-8 was synthesized in an aqueous system using $\mathrm{Zn}\left(\mathrm{NO}_{3}\right) \cdot 6 \mathrm{H}_{2} \mathrm{O}$ as the source for the metal and 2methylimidazole as the organic linker [6]. Electron diffraction was performed in FEI Technai $\mathrm{G}^{2}$ F30 (S)TEM equipped with a Schottky FEG and operated at $300 \mathrm{kV}$. Diffraction patterns were acquired from the nanoparticles at three dose-rates: 2.91, 1.14 and 0.34 e/ $\AA^{2}$ s (Figure 1a). The diffraction patterns were radially averaged for better statistics and a power-law background subtraction was performed to extract the intensities corresponding to the planes. The diffraction intensities were analyzed to obtain the characteristic doses $\left(D_{c}\right)$ for the damage, which is indicated by $50 \%$ reduction in the initial-intensity of the (011) diffraction spots (Figure 1b).

The damage cross-sections obtained for ZIF-8 were then compared with those obtained for MFI zeolite. The threshold values for knock-on damage for $\mathrm{Zn}$ and $\mathrm{N}$ atoms were calculated by using the $\mathrm{Zn}$ Imidazole binding energy of $2.15 \mathrm{eV} /$ bond [8]. The results show that show that knock-on damage should be present at most beam voltages used in typical TEMs. However, we find that radiolysis appears to be the dominating mechanism for damage at $300 \mathrm{kV}$ for MOFs, whereas for MFI, both knock-on damage and radiolysis are active at these energies [9].

References:

[1] H. Furukawa et al., Science 341 (2013), 1230444.

[2] Y. Zhu et al., Nature Materials 16 (2017), 532-536.

[3] D. Zhang et al., Science 359 (2018), 675-679.

[4] R. F. Egerton, Microsc. Res. Tech. 75 (2012), 1550-1556.

[5] O. Ugurlu et al., Physical Review B 83 (2011), 113408.

[6] Y. Pan et al., Chem. Commun. 47 (2011), 2071-2073. 
[7] P. Kumar, Ph.D. Thesis, University of Minnesota, 2018.

[8] R. Gaillac et al., J. Phys. Chem. C 122 (2018), 6730-6736.

[9] This work was supported in part by the NSF MRSEC under award number DMR-1420013, and in part by the Grant-in-Aid program at the University of Minnesota.

a

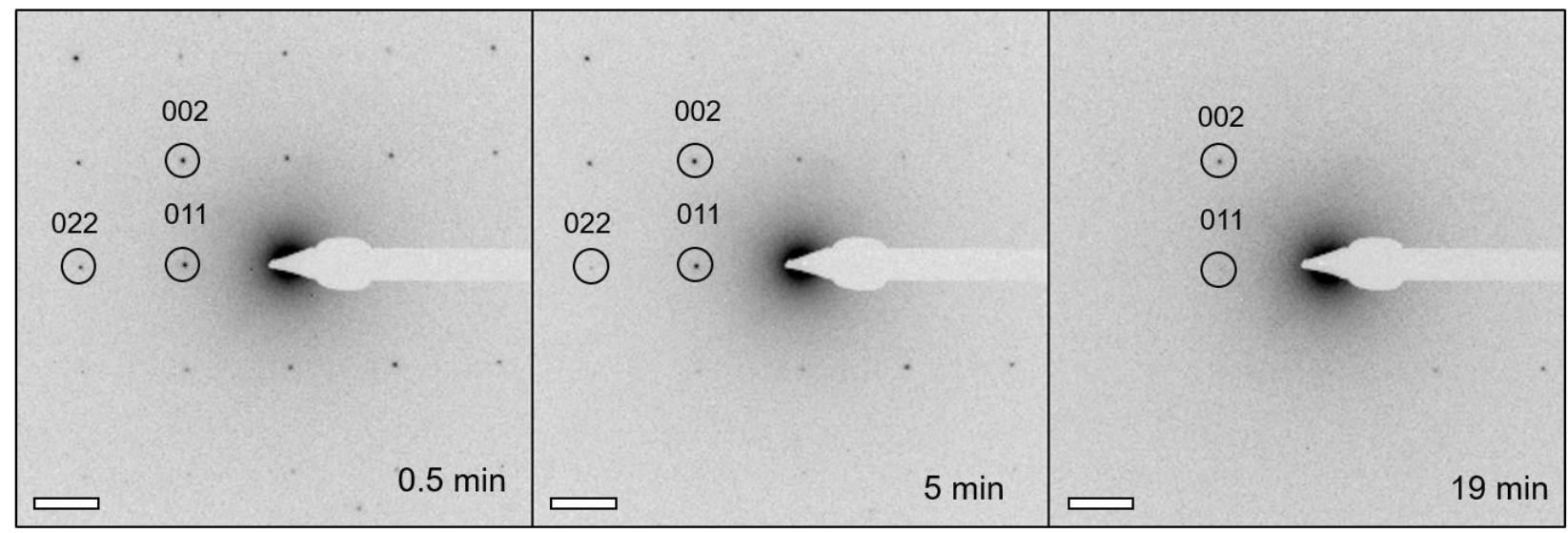

b

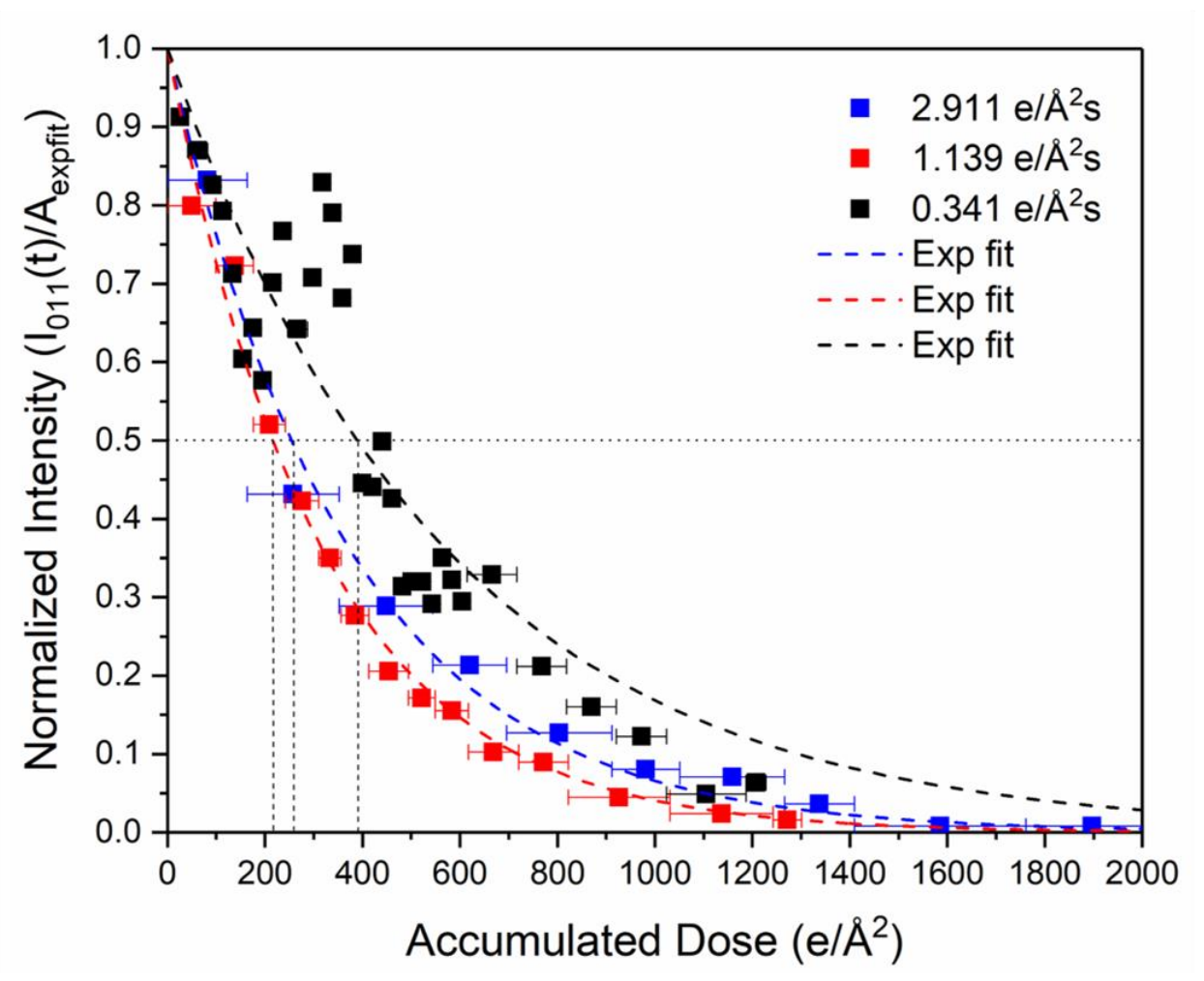

Figure 1: (a) Electron Diffraction collected from a ZIF-8 nanocrystal at beam energy $300 \mathrm{kV}$ and dose rate $0.34 \mathrm{e} / \AA^{2}$, scale bar is $0.5 \mathrm{~nm}^{-1}$; (b) Normalized background subtracted intensities for the (011) spot for the three dose-rates with indicated critical doses $\left(D_{c}\right)$. 